\title{
The Study on the Diffusion of Disinformation via Social Media: How It Happens and Its Effect on Racism Towards Chinese People
}

\author{
Zhengrui Mao, ${ }^{1, *}$, Sitong Song ${ }^{2}$, Weijian Zhou ${ }^{3}$ \\ ${ }^{1}$ Community School of Naples, Naples, 34109, The United States, \\ ${ }^{2}$ School of Media and Communication, University of Leeds, Leeds, LS2 9JT, United Kingdom \\ ${ }^{3}$ UWC Changshu China, Changshu, 215500, China \\ richardmaozhengrui@gmail.com
}

\begin{abstract}
The world has entered the age of social media, which has brought major impact to the human society. We believe it is crucial to consider not only the benefits that social media brought but also the problems. This paper focuses on social media's contribution to the diffusion of disinformation online. This work discusses how the users, social media companies and the fundamental design of the platform supplies the diffusion and leads to the growing racism targeting Chinese people. Acknowledging this issue is the first step to relieving in the long term.
\end{abstract}

Keywords: Social Media, Disinformation, Racism.

\section{INTRODUCTION}

In the era where information and technology are in a central place of everyday life, the social media act as an application of technology to provide information flow. It is vital to keep in mind that all types of information are provided access to spread faster than ever. For example, disinformation on social media can spread at an unbelievable high speed, resulting in other social issues, such as racism. Therefore, it is crucial to understand the fundamental design and algorithm lying behind social media. At the exact moment, users need to be aware of the connection between social media and the effect of diffusing disinformation. Otherwise, the spread of disinformation will turn to growing racism towards Chinese People as it affects people understanding and view of Chinese people based on false information.

The expanding scale and usage of social media provide an opportunity for disinformation to grow, and it is causing the diffusion of disinformation due to users' vulnerability and the biased design of the platform. The result is linked to the growing racist view targeting Chinese people under the influence of political manipulation and algorithm as well as online literacy of media users. This paper will explain how the users and the platforms both contribute to the worsening of the situation and the process of the diffusion worsening racism towards Chinese people.

\section{DIFFUSION OF DISINFORMATION}

\subsection{The Era of Social Media and The Greenhouse for Disinformation}

According to Brian Dean, there are almost 4 billion social media users globally, which is $58 \%$ of the world population [1]. Therefore, it is crucial to understand how social media is contributing to the information we receive. Unfortunately, the information we get on social media is not as neutral as we expect or hope. The diffusion of disinformation is a combined effort of both the platform and the users.

\subsection{Platform Puts Users Into a Tough Spot}

The platform has taken advantage of its users, using users' limited brain powers. Our brains have limited power to process information, and social media had exploited this when they developed the algorithm to analyze what we like and show that preferred information. It has been proven that the companies have the power to know almost exactly what the users enjoy, from political alliances to the favorite sports team. According to Merrill, Facebook identifies your political alliance and feeds personalized political advertisements 
to the users in each category determined by the algorithm [2]. It is easy to see that the information users get when they click "I am feeling lucky" is not entirely up to their luck. This algorithm helps the companies give the users what they wish to see and make sure they continue using their applications. However, users are put into a filter bubble as they are fed the one-sided information they relish viewing. The underlying issue becomes evident: the company keeps the users away from the news that challenges their current view on the world or a particular group of people. While users are being zoned by their preferences and habitual behaviors, it is vital to consider cognitive biases.

\subsubsection{Cognitive Bias}

Cognitive bias is a psychological behavior that suggests people are more willing to share information on risks and dangers and prefer information from people they trust [3]. The imminent result is users paying more attention to the alarming news and the platform utilizing this pattern. When users are in a group with people they know and trust, getting fed about the approaching danger and other threatening news creates noticeable user stickiness and draws significant attention. Furthermore, this is what the companies are doing, with a shady method. The platforms use cookies and logs to analyze our behavior, then put their users into specific groups. When users find out something in their domain, especially the kind about danger, they will find approval and agreement among their communities. The approval will undoubtedly satisfy those behind the screen and keep them focused on whichever app they are using. At the same time, the isolated users are further manipulated and quarantined in their bubbles, and the platform could not care less. Once they stepped into this spot, it is demanding to come clean.

\subsubsection{Inevitable Outcome}

Users hardly get out the biases and filter bubbles because cognitive biases also worsen over time because the tendency to stay with those who share the same belief is tough to correct. When we are given information that contradicts our opinions, we voluntarily search for any evidence that backs our beliefs without merely any notice about the psychological effect. Per the research by Menczer and Hills, after the struggle to prove their own biased point, the bias becomes stronger even if users fail to come up with a logical explanation [3]. The rejection is happening even when the information is neutral. Being trapped by severe cognitive bias is something we naturally would try to avoid. However, our patterns give social media companies the golden opportunity and leverage to consolidate their user stickiness. As users go to social media to seek evidence of their unneutral belief, they step up and give the biased users those information feed that makes them feel supported and confident. It is not their priority to care about whether users see the whole picture. They prefer to focus on pleasing the users necessary, despite that put the users in a jail of their own. The inevitable outcome is that they have made a perfect greenhouse for the spread of disinformation, making users disinformation's prey. Such Platform puts their users in a hard place where they are always being threatened by disinformation. Social media companies should be building a barrier to keep disinformation out of their platforms, rather than opening the gate for them. Users are at imminent risk of being manipulated by disinformation and they are not protecting them enough from the danger.

\subsection{Users Are Vulnerable}

Finishing up with what the platform did, it is crucial to understand how users themselves contribute to the diffusion of disinformation at the same time. Similarly, users put themselves into an undesirable situation because of their limitations. Therefore, their own limits put themselves into a harmful place, making themselves vulnerable. Since many of the users view social media as gateway to accessible information. The rising problem is whether we are getting accurate, correctly portrayed information or twisted ones. If the information we face is not entirely accurate, it is essential to be able to spot the false information. Contradicting to what we believe, we do not have much talent for identifying accurate information among other disinformation. One of the main reasons is the information overload primarily brought to us by social media. Before the age of social media and way back, information is tremendously more sacred because a few controls its access. During the age of attention surplus, people had more than enough time to process and identify each piece of information. The situation changed after social media dominated our internet and lives. Social media has indeed liberated the information, providing access to near everybody. The problem stands being social media creates an information surplus as it brings more than enough information for our brains to process. On this ground, it is unrealistic to think we pay attention to everything we encounter. Despite the problem stands, many of users are unaware of where we are. When users do not pay enough attention to the information they obtain, the spread of disinformation becomes easier, especially with the easy access provided by social media. There is more human limitation that promotes the spread of disinformation.

\subsubsection{Human's Response to Opposing Information}

Among the psychological effects that limits users' ability to identify disinformation, one vital aspect to consider about users' contribution to the spread is the fact that humans are not receptive about opposing information and opinion. The thought process is simple 
and yet challenging to alter. Humans think about what the information means when we first encounter it, but our brain refuses to correct or invalidate our original thought even there is change later [4]. The recent pandemic is an example that illustrates how this pattern functions in real life. Based on the report from Abrams, nearly $30 \%$ of U.S. adults believed that the Chinese government created coronavirus as a bioweapon back in March 2020 [4]. Moreover, in June, about $25 \%$ of the adults are convinced that the virus is purposefully leaked by the people in power. Even some patients who are dying from coronavirus still believe it is a hoax. With the influence of social media, the unprecedented easy access to get and share information enables us to be further jailed by disinformation. When users get disinformation from social media, those who are tricked begin actively hunting information for an unreal idea they have. With social media, they can find more disinformation that supports their idea at the edge of their fingertips. The convinced users later go on their pages and continue to spread the same disinformation they were given. There is virtually no means to get them out of their loops since they search for more disinformation and refuse to take any different idea due to their confirmation and cognitive bias. The identical happening will occur to more users that the social media companies select to be the people that are alike. The spread of disinformation will then infect the users like a virus. In the end, those who are infected still believe the spread is a hoax. It is foreseeable that users' inability to maintain perfectly critical thinking when gathering information on social media will cause the spread of disinformation.

\section{THE FLAWED AND BIASED DESIGN OF PLATFORM}

Apart from the deliberate contribution by the users and social media companies, there is also noticeable aid from the fundamental design of social media platform that pushes the diffusion of disinformation inadvertently. Social media initially was not created for the spread of misinformation or disinformation, like the dynamite invented by Nobel, they were designed with successful achievement on their original goals [5]. However, further potential development and pragmatic execution would largely be influenced by executives and lead to unexpected impacts.

\subsection{Potential Problems in Algorithms}

As the popularization of social media going on, the deployment of the algorithm also widely spreads out. Facebook finds user's prospective friends according to their personal profile. YouTube recommends videos that users may be interested in based on their searching and watching history. Via algorithms, multitudes of the repeatable process are executed more efficiently and cost-saving. As a process in calculating and problem- solving, the algorithm is always a black box, an opaque machine that takes inputs and carries out some inscrutable process and delivers unexplained outputs based on that process [6]. The undisclosed process is hardly understood and always treated as confidential by the entities that use it [7]. Therefore, users are possibly excluded from information that they may be potentially desired to know passively. More importantly, such a complex and opaque process would be difficult to supervise and correct, especially while encountering the following flaws [8]. Firstly, matching systems could poorly designed, which is required to provide information index function. For example, when social media is relying on an algorithm on recommended content, the matching system may result in biased information because of blind spots or outdated mechanisms. Secondly, correlations were assumed in decision-making systems, which are personally tailored by the biased programmer/executive because of the frequent mutual occurrence of two elements. For example, a programmer might relate the spread of COVID19 with Chinese, which should be a causal relationship between them, leading to discriminatory content. Lastly, the dataset being used is inappropriate, which leads to an inevitable skewed algorithmic system that effectively outputs discrimination because of the flawed initial inputs. For example, due to the information unavailability, in some areas, data that meet the standard are rare. Thus, this portion of data would be overrepresenting in these areas, causing some patterns of discrimination.

\subsection{Eventual Further Spread of Disinformation}

Each defect above could lead to the further spread of disinformation with users' trust and users' unawareness. The imperfect matching systems could be problematic and deliver disinformation or misinformation, leading to bias. For instance, the alt-right, one far-right organization in the U.S. used a variety of techniques to trick the algorithm and push the spread of disinformation and propaganda in Google's searching result ranking [9]. Similarly, the pre-assumed correlation by social media cultivates the bias to users. According to the research by progressive research center Media Matters, New York City's television over-assumed the involvement of black in crimes [10]. The suspect rate of black criminals far exceeded the actual arrest rate of black for crimes [10]. The results of the algorithm from incognizant flawed data sets conveying race-discriminatory ideas are also typical. In 2017, Apple's Face ID system was not able to distinguish two Chinese faces as accurately as how it did with samples pulled from western countries [11]. The deficiencies of extensive-used algorithms create harmful content and promote the spread of bias in a massive population of users. 


\subsection{Further Limitation of Moderation}

Moreover, to ensure content in appropriate values and platform's intentions and against the consequences of improper design mentioned above, most social media have set content moderator teams to address these inappropriate contents organizationally and systematically. However, the incidents continue. In given cases, what prevents social media platforms from effectively alleviating disinformation, misinformation, and bias [12]? There are multiple factors addressing these issues. Firstly, the scale of social media nowadays is gigantic and unprecedented. Social media is now on the way to dominate everyone's life, as more than half of the world is now using social media. Facebook is in the leading position, having 2.7 billion monthly active users, followed by YouTube ( 2 billion), WhatsApp ( 2 billion), Facebook Messenger (1.3 billion), and WeChat (1.2 billion). Among all internet users, $83.36 \%$ of users involved in social media. According to YouTube, people around the world watch over a billion hours of video every single day. Twitter has 139 million daily active users, who send more than 500 million tweets per day [1]. Behind all these numbers is the information flow that has never been seen in human history. Facing such a vast number, the content moderating efficiencies of limited labor under each social media company are undoubtedly not enough. The lack of comprehensive moderation on content certainly leaves content that is not with appropriate value. Beyond that, moderation would also arouse the disputation on freedom of speech. It is true that manual content moderation is the most proper disposal in terms of the correctness of the content. Nevertheless, as mentioned above, the magnanimity of information on social media platforms vetoes the possibility of manual regulation, considering the manual cost and efficiency. The tremendous work stress is making this job the most undesirable working experience ever, according to Weber and Seetharaman [13]. Hence, the moderating vocations are mostly on algorithms. By deploying specific artificial intelligence, which has particularly trained in this direction, social media platforms could save money and censor the content to some extent. When it comes to Artificial Intelligence (A.I.) content moderation, A.I. not being capable of understanding the context, nuance, and connotation is a problem that persists. This problem puts the platform into a dilemma, reporting innocuous contents or missing undesirable contents [12]. Both have severe defects. False positives would arouse users to protest content moderation system. And false negatives would leave harmful content on social media. Under the current circumstances, content moderation on social media is still an unsolvable issue no matter via manual work or via algorithm. Therefore, false information can spread through holes that have not been patched, because of the dilemma of moderation system.

\section{DISINFORMATION'S EFFECT ON RACISM TOWARDS CHINESE PEOPLE}

\subsection{Diffusion to Racism}

During the diffusion and consumption of information on social media platforms, the constantly occurring and increasing racism is a problem to be concerned. This is mainly reflected in people of color, including Chinese people. The case study here focuses on the disinformation and racism to Chinese. Since the outbreak of the coronavirus at the beginning of 2020, Chinese people have been stigmatized and endured more racial discrimination than before the pandemic. In a hate speech analysis about Twitter, Reima Al-Jarf argues that COVID-19 contributed to the aggravation and rise of Sinophobia over the internet [14]. Moreover, it can be observed that the discrimination brought by the pandemic has contributed to forming a deeper racial bias with the aid of social media [15]. Asian Americans have been constantly portrayed as outsiders rather than accepted as Americans, excluded from the American community [15]. With the observation above, it is fair to argue that social media is responsible for contributing to the rise of racism online. It is argued earlier in this paper that algorithm helps companies to form filter bubbles, creating obstacles for social media users to acquire information that challenges their existing views. This is a crucial reason for the dissemination of disinformation and hate speech targeting Chinese people. Social media companies have dominating power over what information is the users exposed to, and this is done when the users are given false ideas about their own research. Then, companies have molded social media to satisfy users about all their views of the world, regardless of whether they are biased or not. The current moderation or regulation of the spread of disinformation is not effective enough to control the current happening. The result can be devastating. The false information is often greatly capable of drawing attention as they frequently satisfy viewers' need, although they are not valid. The priority of the companies will worsen the spread of disinformation on social media. Biases and hate speech are effective for drawing attention and engagement, and people are easily misled by this type of information, leading to misunderstanding and potentially racism against Chinese and possibly other Asians. Additionally, the impact of online racism towards Chinese due to Covid-19 will be discussed in terms of political propaganda and users' critical views.

\subsection{Factors that Contribute to The Growing Racism}

\subsubsection{The Influence of Politics}

From a political perspective, the spread of racism online can be understood as a way of political 
propaganda and manipulation. Being more than a platform for public opinion expression, social media also act as a tool to manipulate personal and public opinion. Woolley and Howard argue that social media are utilized for social control in authoritarian countries, and in democratic societies they serve as tools for computational propaganda by manipulating public propositions [16]. Social media technically do not control user's behaviors and thoughts because as a tool they are used by people to achieve goals, not vice versa. However, when politicians utilize these tools with a political purpose, they are given the role of political propaganda, and in that way, they have impact on public opinions [17]. Therefore, In whatever sort of political environment, social media are found to be an effective tool for political propaganda. Donald Trump can be an example of this. As an overtly biased speech being released, Trump used to call the coronavirus 'Chinese Virus.' It is not sure whether the former U.S. president was deliberately guiding hatred towards Chinese people. Nevertheless, there still appear consequences. Research shows that the week after he first tweeted these words witnessed an increase in hate crimes targeting Asian Americans and anti-Asian hashtags [18]. We suppose specific purposes for him to tweet these contents, such as the rage caused by racism, lead to hatred of Chinese, and then the immigration rate might be reduced. On the other hand, the biased speech arouses the awareness of antiracism. Hashtags such as \#StopAsianHate begin to appear on Twitter and Instagram, and they receive attention. People did not care about what Asians were going through before President Trump was accused of a spreader of racism as much as they care about it now. The racism announced by politicians indeed harms the equality among ethnicities, but it also brings the issue to a global stage to be focused on and considered.

\subsubsection{The Influence of Online Literacy and Algorithm}

Besides political factors, the public's reaction towards racist content on social media also determines how Chinese people are influenced by racism. As a way for communication with open access, social media provide more opportunities for users of identical negative ideas to gather with technology serving as a catalyst [19]. When racists are organized as a group, what they believe and promote will affect not only their own kind but also other people outside of the group. For racists, being together and sharing the same ideas means having a sense of being recognized and affirmed. For other people, a media environment filled with negativity will gradually assimilate them. Those ideas are similar to toxic: they negatively change people's thoughts and cause firm belief. However, considering from the perspective of media users, their ability to judge and think matters more. In other words, their literacy of information in this digital era is in an important position. Literacy varies from user to user, which means their capacity of dealing with information decides how they react to biased content, disinformation, and misinformation on social media. For example, there used to be many rumors about the origin of the coronavirus. One of them claimed by a famous mystifier Uri Geller is that the coronavirus was released intentionally from a Chinese military lab, aiming to destroy the global economy [20]. To verify if this is disinformation is not complicated. There are reports of the genetic analysis indicating that it is from nature, and they are available online if people want to read them. The problem is that most of the audiences will not critically think about the veracity of this information as they see it, and they probably do not even realize this could be disinformation, resulting in further spread of it and more discrimination. Also, algorithms tend to feed people with the information, which will provoke rage and fear and attract more user engagement [19]. In other words: irritant messages spread faster than the 'boring' truth. Even if some people are not involved in the diffusion of disinformation, their voices will eventually be covered by those contents with a greater value of gaining attention. We believe the attention-driven algorithm expands the influence of disinformation, and since most people are not perfectly literate to think of and question that disinformation, racism still exists.

\section{CONCLUSION}

It is easy to see that humanity has walked into the era of information and technology. Social media has set its foot on dominating people's access to information. Knowing social media companies exploiting users' weaknesses and users' own limitations, it is factual that social media is aiding the diffusion of disinformation, especially when the fundamental design of social media platforms is biased and flawed. The imminent result is the growing racism targeting Chinese people as the spread of disinformation is under the influence of political propaganda and existing racism among the users.

It is vital for people to understand the fundamental idea of the diffusion of disinformation via social media. It is only possible to contain the spread after users become aware of this contagious issue. Although there is no great cure for the problem at this moment, we hope the situation will be relieved after the world recognizes the issue and approach it with caution.

\section{REFERENCES}

[1] Dean, B. (2021). How Many People Use Social Media in 2021? https://backlinko.com/socialmedia-users.

[2] Merrill, J. B. (2016). Liberal, Moderate or Conservative? See How Facebook Labels You. https://www.nytimes.com/2016/08/24/us/politics/f 
acebook-ads-politics.html.

[3] Menczer, F., \& Hills, T. (2020). Information Overload Helps Fake News Spread, and Social Media Knows

It.

https://www.scientificamerican.com/article/inform ation-overload-helps-fake-news-spread-and-socialmedia-knows-it/.

[4] Abrams, Z. (2021). Controlling the spread of misinformation. Monitor on Psychology, 52(2). http://www.apa.org/monitor/2021/03/controllingmisinformation

[5] Srinivasan, R. (2019). Questioning Connectivity. In Beyond the valley: how innovators around the world are overcoming inequality and creating the technologies of tomorrow. The MIT Press, Cambridge. pp. 181-190, 25.

[6] Pasquale, F. (2015) The Black Box Society: The Secret Algorithms That Control Money and Information. Harvard University Press, Cambridge.

[7] Dwork, C. and Mulligan, D. (2013) "It's Not Privacy, and It's Not Fair." 66 Stan. L. Rev. Online 35.

http://www.stanfordlawreview.org/online/privacyand-big-data/its-not-privacy-and-its-not-fair.

[8] United States, Congress, Executive Office of the President. Big Data: a Report on Algorithmic Systems, Opportunity, and Civil Rights

[9] Solon, Olivia. (2016) "How Google's Search Algorithm Spreads False Information with a Rightwing Bias." www.theguardian.com/technology/2016/dec/16/go ogle-autocomplete-rightwing-bias-algorithmpolitical-propaganda.

[10] Angster, Daniel. (2015) "Video: What Happens When Local News Over-Represents AfricanAmericans As Criminals." www.mediamatters.org/legacy/video-whathappens-when-local-news-over-represents-africanamericans-criminals.

[11] Zhao, Christina. (2017) "Is the iPhone racist? Apple Refunds Device that Can't tell Chinese People Apart." http://www. newsweek.com/iphone-x- racist-apple-refundsdevice- cant-tell-chinese-people-apart- woman$751263 /$.

[12] Etlinger, Susan, and Centre for International Governance Innovation. (2019) Models for Platform Governance. Report. http://www.jstor.org/stable/resrep26127.6.

[13] Weber, Lauren, and Deepa Seetharaman. (2017) "The Worst Job in Technology: Staring at Human Depravity to Keep It Off Facebook." www.wsj.com/articles/the-worst-job-intechnology-staring- at-human-depravity-to-keep-itoff-facebook-1514398398.

[14] Reima Al-Jarf. (2021) Combating the Covid-19 Hate and Racism Speech on Social Media. Technium Social Sciences Journal, 18(1). https://doi.org/10.47577/tssj.v18i1.2982

[15] Darling-Hammond, S., Michaels, E. K., Allen, A. M., Chae, D. H., Thomas, M. D., Nguyen, T. T., Mujahid, M. M., \& Johnson, R. C. (2020) After "The China Virus" Went Viral: Racially Charged Coronavirus Coverage and Trends in Bias Against Asian Americans. Health Education \& Behavior, 47(6), 870-879. https://doi.org/10.1177/1090198120957949

[16] Woolley, S. C., \& Howard, P. N. (2019) Computational propaganda: political parties, politicians, and political manipulation on social media. Oxford University Press. https://doi.org/10.1093/oso/9780190931407.001.0 001

[17] Romer, D., \& Jamieson, K. H. (2020) Conspiracy theories as barriers to controlling the spread of COVID-19 in the U.S. Social Science \& Medicine, 263 , 113356. https://doi.org/10.1016/j.socscimed.2020.113356

[18] Reja, M. (2021) Trump's 'Chinese Virus' tweet helped lead to rise in racist anti-Asian Twitter content: Study. https://abcnews.go.com/Health/trumps-chinesevirus-tweet-helped-lead-riseracist/story? $\mathrm{id}=76530148$

[19] Cohen, S. B. (2019) Perspective | The 'Silicon Six' spread propaganda. It's time to regulate social media sites. https://www.washingtonpost.com/outlook/2019/11 /25/silicon-six-spread-propaganda-its-timeregulate-social-media-sites/.

[20] Bosotti, A. (2021) Uri Geller sparks angry backlash as he claims Covid was 'intentionally released' https://cn.bing.com/search?q=coronavirus+release $\mathrm{d}+\mathrm{by}+$ China \& go $=$ Search $\& \mathrm{qs}=\mathrm{n} \&$ form $=\mathrm{QBRE} \& \mathrm{sp}$ $=-1 \& \mathrm{pq}=$ coronavirus + released $\& \mathrm{sc}=0$ 20\&sk=\&cvid=3AAE6CEF4123443F8C61D3E79 24446D9 